\title{
Gemüse gegen Fleisch, Altchinesische Ernährungslehre und Yang-Mangel - eine Analyse statistischer Trugschlüsse in einer Ernährungsstudie
}

\author{
Johannes G. Schmidt \\ Stiftung Paracelsus heute, Einsiedeln, Schweiz
}

\section{Schlüsselwörter}

Ernährung · Fleisch - Tierische Eiweisse - Statistische Trugschlüsse . Confounding Bias · Altchinesische Medizin · Feuerschule · Yang-Mangel

\section{Zusammenfassung}

Von einem Verlag für chinesische Medizin wurde uns im Herbst 2010 eine Studie in Buchform angeboten, die beweisen soll, dass Fleisch ungesund sei und Herzinfarkt und Krebs erzeuge, wogegen Gemüse und Ballaststoff diese Krankheiten verhindere. In der Altchinesischen Ernährungslehre allerdings ist Fleisch ein Fundament der Ernährung und schützt als wichtige Yang-Quelle vor Krankheiten. Eine Analyse der wissenschaftlichen Evidenz dieser Studie lässt denn auch bekannte statistische Trugschlussmöglichkeiten erkennen, die falsche Ergebnisse vortäuschen können. Hinter Symptomen von falscher Hitze erkennt die Altchinesische Medizin den Yang-Mangel, etwa bei menopausalen Beschwerden. Wird falsche Hitze durch Yin-Arzneien «kaltgestellt», nehmen Krankheitsprobleme auf Dauer zu. In der Tat erhöht sich die Sterblichkeit unter der Yin-Tonifikation mit Östrogenen. Im gleichen Sinne zeigt sich, dass auch antioxidativ wirkende Vitaminbehandlungen zu einer Zunahme von Krankheiten führen. Geschwächtes Yang bedeutet grössere Anfälligkeit für Krankheiten. Fleisch nährt das Yang, und Menschen mit schwachem Yang tendieren deshalb zu mehr Fleischkonsum. Menschen mit Yang-Schwäche bekommen nicht wegen, sondern trotz des Fleischkonsums häufiger Krankheiten.

\section{Keywords}

Nutrition · Meat · Animal proteins · Statistical fallacies .

Confounding bias · Ancient Chinese medicine · Fire school . Yang deficiency

\section{Summary}

Vegetables against Meat, Traditional Chinese Dietetics and Yang Deficiency - an Analysis of Bias and Statistical Fallacies in a Dietary Study In the autumn of 2010, a publisher of Chinese medicine advertised a book based on a dietary study conducted in China pretending to prove that meat is unhealthy and leads to cardiac infarction and cancer whereas vegetables protect against these diseases. In Ancient Chinese medicine, however, meat is a basic component of nutrition and constitutes the most important source of Yang which enables a person to resist diseases. The analysis of the scientific evidence of this study indeed reveals well-known statistical fallacies and biases that may confound the alleged findings. In Ancient Chinese medicine, symptoms of false heat are considered to be the result of Yang deficiency, as in the case of menopause disorders. If false heat is treated with Yin medicines, Yang declines and, thus, the occurrence of diseases may rather increase. Yin tonification by the use of estrogens, as has in fact been shown, leads to an increase of total mortality in postmenopausal women. Similarly, treatment with antioxidant vitamins also compromises Yang and can lead to an increase of diseases. A compromised Yang means an added susceptibility to diseases. Meat is an important dietary source of Yang, and therefore people with Yang deficiency may well tend to eat more meat. As a result, people with a weak Yang may suffer more from diseases not because, but in spite of the consumption of meat.

\section{KARGER

Dr. med. Johannes G. Schmidt

Stiftung Paracelsus heute

Praxiszentrum Meinradsberg

Ilgenweidstrasse 3, 8840 Einsiedeln, Schweiz

Tel. +41 55-4188191, Fax -4188192

schmidt@paracelsus-heute.ch 


\section{Mots-clés}

Nutrition - Viande · Protéines animales · Erreurs statistiques . Biais d'erreur - Médecine traditionnelle chinoise - École du feu . Manque de Yang

\section{Résumé}

Les légumes contre la viande, la diététique traditionnelle chinoise et le manque de Yang: analyse des erreurs statistiques dans une étude de nutrition

En automne 2010, une maison d'édition de Médecine chinoise nous a proposé un livre rapportant une étude censée prouver que la viande nuit à la santé et engendre des crises cardiaques et le cancer, alors que les légumes et les fibres alimentaires empêchent ces maladies. Selon la diététique traditionnelle chinoise, la viande constitue cependant une base de la nutrition et protège des maladies en tant que source importante de Yang. Une analyse des données scientifiques de cette étude a aussi mis en évidence d'éventuelles erreurs statistiques bien connues pouvant fausser les résultats. Selon la Médecine traditionnelle chinoise, les symptômes de fausse chaleur révèlent le manque de Yang, comme lors de troubles de la ménopause. Si la fausse chaleur est «rafraîchie» grâce à des remèdes Yin, les problèmes de santé augmentent de manière durable. En fait, la mortalité est accrue lorsque le Yin est tonifié par des œstrogènes. Dans ce sens, il a été montré que des traitements à base de vitamines antioxydantes entraînent une augmentation des maladies. Un affaiblissement du Yang signifie une prédisposition accrue aux maladies. La viande renforce le Yang et les personnes dont le Yang est faible ont donc tendance à consommer plus de viande. Les personnes dont le Yang est faible tombent plus souvent malades non pas à cause de la consommation de viande, mais malgré elle.

\section{Einführung}

Vom «Verlag für Ganzheitliche Medizin» (Bad Kötzting, Deutschland), einem Verlag für chinesische Medizin, wurde uns im Herbst 2010 eine Neuerscheinung angeboten, die unter dem Namen China Study «verblüffende Konsequenzen für die Lebensführung» anpreist. Der Nachweis sei erbracht - so die US-amerikanischen Autoren -, tierische Eiweisse seien ganz ungesund und Gemüse und Ballaststoff hingegen sehr gesund. In wissenschaftlichem Format veröffentlicht wurde die "Cornell China Study» bereits im Jahr 1998 im American Journal of Cardiology, damals noch mit chinesischen Koautoren [1].

In der Altchinesischen Ernährungslehre gibt es das Gleichnis vom Mauerbau: Fleisch sei wie der Stein, Getreide wie der Mörtel, Gemüse wie der Verputz, Früchte wie die Farbe. Wie in diesem Bild zum Ausdruck kommt, braucht es gewisse Proportionen zwischen diesen Bestandteilen, damit die Mauer bzw. die Hauswand kräftig, tragend, schützend und schön ist. Das Bild zeigt auch, dass
Mauern je nach Ort und Funktion etwas verschieden sein müssen und verschiedene Zwecke erfüllen, so wie auch Menschen interindividuell verschieden sind und manchmal unterschiedliche Ernährungsschwerpunkte benötigen. Verputz und Farbe allein sind sicherlich zu wenig, damit eine Mauer stabil und «gesund» ist. Mit Steinen und Mörtel hingegen hält eine Mauer, mit Verputz und Farbe wird sie abgerundet und schön. Also sind Gemüse und Früchte allein zu wenig. Etwa eine Hälfte Getreide, ein Drittel Gemüse und ein Sechstel Fleisch bilden eine ausgewogene Ernährung. Fleisch (im Mass) ist also nach diesem Bild der Altchinesischen Ernährungslehre ganz generell Fundament einer guten Ernährung, wenn auch nicht alle Fleisch im gleichen Ausmass nötig haben. Auch die klassische Ayurveda-Heilkunst lehrt keinen Vegetarianismus - entgegen verbreitetem Halbwissen. Nach der ayurvedischen Ernährungslehre ist der Verzehr von Fleisch besonders nötig bei ausgezehrten Menschen und Menschen mit einer bestimmten schwachen Konstitution.

Die angeblichen Studienergebnisse und die vegane Stossrichtung des von diesem Verlag für chinesische Medizin angepriesenen Pamphlets liegen also quer zum alten Wissen der chinesischen Ernährungslehre. Wir wollen anhand einiger methodischer Regeln wissenschaftlicher Evidenz die Aussagekraft der China Study a nalysieren und Yang-Schwäche und Yang in der Nahrung als möglichen Hintergrund (Confounder) der möglichen Trugschlüsse dieser Studie untersuchen.

\section{Evidenz und bekannte Formen statistischer Trugschlüsse}

Die moderne Propaganda krankheitsvorbeugender Mittel und Massnahmen, die vor Krankheit bewahren und das Leben verlängern sollen, einerseits, ebenso wie Warnungen vor ungesunden Dingen andererseits haben heutzutage ein verwirrendes Ausmass erreicht. Argumentiert wird jeweils mit wissenschaftlichen Studien, die angeblich den Beweis für die propagierten Medikamente, Produkte und Massnahmen lieferten. Sehr oft werden dabei Studienergebnisse über deren Geltungsbereich hinaus überinterpretiert und/oder es werden bekannte statistische Trugschlüsse übersehen. Es gibt Schätzungen aufgrund einer methodisch sorgfältigen Evidenzbeurteilung, wonach sogar bei anerkannten medizinischen Massnahmen und Empfehlungen der sogenannten Schulmedizin in der grossen Mehrheit nicht schlüssig ist, ob diese wirklich nützen oder vielleicht eher schaden.

Die China Study ist eine (ökologische) Querschnittsstudie [1], also eine Beobachtungsstudie, und keine kontrollierte Vergleichsstudie. Üblicherweise werden Risikofaktorenstudien als Kohortenstudien (Verlaufsstudien) angelegt, damit Klarheit besteht über die Zeitabfolge zwi- 
schen Risiko und Folge [2]. Schon dies kann die China Study nicht leisten. Zudem limitiert der Vergleich von «ökologischen Einheiten» statt von Individuen die Aussagekraft, denn wir wissen nur, dass ein Unterschied zwischen Ernährung und Krankheitshäufigkeiten zwischen den USA und dem ländlichen China besteht, aber keineswegs, ob dieser Unterschied auch zwischen ländlichen Chinesen besteht, die Fleisch essen, und solchen, die kein Fleisch essen. In ihrer Veröffentlichung im American Journal of Cardiology halten die Autoren noch fest, «für die Beurteilung des Krankheitsrisikos von Individuen brauche es eine andere Studie» [1].

Doch selbst wenn die Ergebnisse aus einer Kohortenstudie (mit gut definierter Inzeptionskohorte) kämen, hätten wir immer noch keine kontrollierte Studie. Die im Folgenden genannten statistischen Trugschlüsse gelten für Querschnitts- wie für Kohortenstudien, die beide «nur» Beobachtungsstudien (ohne Kontrollgruppe) sind [2]. «Kontrolliert» ist eine Studie, wenn bekannte und möglichst auch unbekannte Faktoren (Confounder) in den Vergleichsgruppen so gleichmässig verteilt sind (z.B. nach dem Los), dass sie den Vergleich nicht beeinflussen bzw. verfälschen oder stören können (Confounder werden auf Deutsch auch «Störfaktoren» genannt). Dieses Trugschlusspotenzial in den vielen nichtkontrollierten Studien liefert dann auch die einfache Erklärung, weshalb aus der Ernährungsforschung immer wieder derart viele falsche und sich widersprechende "Erkenntnisse» vorgebracht worden sind. Auch die offizielle Ernährungswissenschaft der Schulmedizin ist deshalb voll von Spekulationen, die geradeso gut falsch wie richtig sein können.

Dazu kommt in vielen Studien die Möglichkeit eines Surrogat-Fehlers (Surrogat-Trugschluss), wenn nicht die Auswirkungen auf das Leiden des Patienten selbst, sondern nur die Auswirkungen auf seine pathophysiologischen Messwerte erfasst worden sind. Die praktische Anwendung wissenschaftlicher Studienergebnisse wird überdies erschwert durch die fehlende individuelle Prädiktion beim einzelnen Patienten, denn Studienergebnisse sind Durchschnittsergebnisse [3]. Und die «number-needed-to-treat» ist dabei fast regelmässig hoch, sodass selbst die Ergebnisse methodisch guter Studien - vor allem im Bereich der Risikominderung und der Prävention - praktisch und klinisch irrelevant sein können und für den einzelnen Menschen/Patienten keine vernünftige Aussagekraft haben [4].

\section{Confounding Bias}

Wenn wir beispielsweise den Unterschied zwischen Äpfeln und Birnen nicht kennten, würde uns eine Analyse von Merkmalen zeigen, dass die rote Farbe mit der runden Form der Kugel eine Korrelation aufweist - oder die grüne Farbe mit einer langgezogenen Glockenform. Rotes Obst (Äpfel

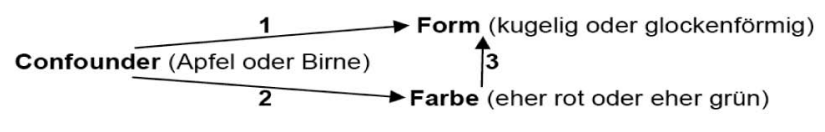

Abb. 1. Confounding-Fehler (confounding bias): 1 = wahrer Zusammenhang, 2 = Confounding, 3 = Scheinzusammenhang (Epiphänomen).

und Birnen vermengt) ist überhäufig kugelförmig, grünes Obst überhäufig glockenförmig. Also zeigt diese Analyse scheinbar, dass die rote Farbe eine Kugelform des Obstes verursacht und die grüne Farbe eine Glockenform. Denn Birnen sind meist glockenförmig und selten rot; Äpfel hingegen sind rund und haben oft eine rote Farbe. Aus Unwissenheit, dass man Obst in Äpfel und Birnen unterteilen kann, folgert man also vorschnell, dass die Farbe die Form bestimme, obwohl tatsächlich die Form von der Obstart bestimmt wird - Apfel oder Birne. Man hat aus Unkenntnis «Äpfel mit Birnen verglichen». Die Farbe ist nur ein Epiphänomen (Scheinzusammenhang), konfundiert durch den wahren Zusammenhang zwischen Obstart und Form. Diesen Trugschluss nennt man ConfoundingFehler (confounding bias) (Abb. 1).

Der Confounding Bias wird als Selection Bias bezeichnet, wenn das Confounding durch einen erkennbaren Selektionsvorgang zustande kommt. In die Gruppe des roten Obstes sind - im Beispiel - überhäufig Äpfel (und nicht Birnen) selektioniert worden. Diese Selektion führt zum Confounding durch die Obstart und zum gezeigten Scheinzusammenhang. Unter dem Begriff Selection Bias kennen wir seit 20 Jahren viele Beispiele, bei denen vorschnelle und unkritische Interpretationen aus Verlaufsund Querschnittsstudien so sehr in die Irre geführt haben, dass ein klarer Nutzen eines Faktors gefolgert worden ist, wo in Wirklichkeit ein Schaden vorgelegen hat $[5,6]$.

Von den vielen Beispielen ist die sogenannte Hormonersatztherapie in der Menopause/Postmenopause aufschlussreich. Aufgrund einer deutlichen statistischen Korrelation zwischen der Östrogeneinnahme und dem verminderten Auftreten von Herzinfarkten in Kohortenstudien wurde lange behauptet, die Hormonersatztherapie würde zu einer deutlichen Verminderung von Herzinfarkten führen; Östrogene sollten deshalb unbedingt von allen Frauen präventiv eingenommen werden. Eine später begonnene kontrollierte Studie (Women's Health Study mit zwei durch das Los, d.h. Randomisation, hergestellten identischen Vergleichsgruppen) musste dann aber nach 5 Jahren vorzeitig abgebrochen werden, weil die Frauen unter Östrogentherapie signifikant häufiger starben als die Frauen in der unbehandelten Kontrollgruppe. Von einer Senkung der Herzinfarktrate keine Spur [6]. Wie kommt das? In den früheren Studien täuschte ein Selection Bias diesen scheinbaren Nutzen vor, wo in Wahrheit eine schäd- 
liche Wirkung der Östrogentherapie vorhanden war. Einige Autoren hatten diesen Bias postuliert und auf den möglichen Fehler aufmerksam gemacht $[7,8]$. Solche statistischen Fehler können also sogar das Gegenteil dessen vortäuschen, was wahr ist. Frauen, die Hormone einnahmen, waren nämlich eine Selektion derjenigen, die von ihren Ärzten als frei von kardiovaskulären Risiken beurteilt worden waren, denn kardiovaskuläre Risiken waren damals eine Kontraindikation für die Verschreibung von Östrogenen. Umgekehrt befanden sich unter den Frauen ohne Hormoneinnahme ja all diejenigen, die kardiovaskuläre Risiken aufwiesen und deshalb keine Östrogene bekommen hatten. Dazu gibt es weitere Selektionseffekte (z.B. healthy complier bias), die wir hier nicht weiter ausführen wollen.

\section{Altchinesische Medizin}

Wie betrachten und behandeln wir Menopausenbeschwerden wie Wallungen/Schwitzen und Schlafstörungen in der Altchinesischen Medizin? Es ist Yang-Mangel! Durch Kälte bzw. Yang-Schwäche läuft der Körper heiss; es ist eine falsche Hitze! Wird falsche Hitze durch Yin-Arzneien gekühlt bzw. «kaltgestellt», dann können in der Tat die Beschwerden oft gelindert werden. Die potenteste Yin-Arznei in diesem Fall sind Östrogene, aber auch kühlende Mittel wie Soja und Cimicifuga (Silberkerze) können Linderung bringen. Mit dieser Symptomtherapie wird der zugrunde liegende Yang-Mangel aber weiter verstärkt, sodass der altchinesische Arzt vermuten muss, dass damit Krankheitsprobleme auf Dauer eher zunehmen. Das Kühlen falscher Hitze (mindestens, ohne gleichzeitig das Yang zu bewahren) ist Ausdruck ungeduldigen Unwissens, und der altchinesische Arzt sieht seine Befürchtungen durch die Ergebnisse der oben erwähnten Women's Health Study bestätigt: Die Sterblichkeit nimmt unter der Yin-Tonifikation mit Östrogenen zu [6].

Natürlich gibt es Formen der Menopausenbeschwerden, die spontan wieder in Ordnung kommen und sich nach der durch die Umstellung verursachten passageren YangErschöpfung erholen, auch wenn solche Frauen eine Zeit lang mit Östrogenen (oder TCM-Rezepturen zur Behandlung des vermeintlichen Yin-Mangels) gekühlt worden sind. Jeder kennt aber auch die Patientinnen, die nach einer vielleicht anfänglichen Verbesserung der Menopausenbeschwerden durch die kühlenden Yin-Mittel nie mehr von ihren Wallungen oder den Schlafstörungen wegkommen. Das mangelnde Yang erholt sich nicht mehr. Wenn wir menopausale Beschwerden von vornherein mit Yang aufbauenden Rezeptstrategien behandeln, dauert es vielleicht etwas länger, bis die Beschwerden verschwinden, aber auch schwere Fälle reagieren mit der Zeit (es kann dann 2 oder 3 Jahre dauern). Diese Behandlung ist nachhaltig im Sinne einer restitutio ad integrum und wirkt so vorbeugend auf neue Krankheiten.

Und natürlich spielt auch die Frage der Dosis eine wichtige Rolle. Ausgewogene Mengen wirken anders als unausgewogene Mengen. Schütten wir etwas Schnaps (oder Fett oder Zucker ...) ins Feuer, dann brennt es besser und brutzelt oder riecht fein nach Karamell. Schütten wir aber viel Schnaps (oder viel Fett oder Zucker ...) ins Feuer, dann ersticken wir das Feuer, und es erlischt. Stetige Dosis-EffektBeziehungen gibt es also in der Natur nur in selektiven Beobachtungsabschnitten und nicht generell. Wenn eine Studie eine lineare Dosis-Effekt-Beziehung aufgezeigt haben will (wie die China Study), dann sind methodische Fehler zu vermuten (oder die Ergebnisse sind nur selektiv gültig und lassen sich nicht verallgemeinern).

Nehmen wir bei zu schwachem Verdauungsfeuer viele Vitamine ein, dann schwächt/kühlt die antioxidative Wirkung die Verdauung; die Vitamine werden nicht «verbrannt», sondern blockieren gewisse Symptome (was eine trügerische Verbesserung bringt) und fehlen dem Körper dort, wo er sie brauchen würde. In der Tat zeigt sich, dass antioxidative Vitaminbehandlungen zu einer Zunahme von Krankheiten führen können [9, 10]. Nehmen wir hingegen bei gutem Verdauungsfeuer nur die wenigen Vitamine aus der normalen Nahrung zu uns, dann «verbrennt» die Verdauung die wenigen Vitamine, und der Körper hat genug davon. Ob wir genug Vitamine, Eisen usw. haben, hängt also davon ab, dass wir das Verdauungsfeuer stärken (Yang «kocht» das Blut und macht es rot), und nicht davon, ob wir viel oder wenig einnehmen oder gar infundieren (Infusionen kühlen das Yang stark ab - vor allem im Winter; damit wird die Yang-Schwäche als Grund des «Eisenmangels» erst recht chronifiziert).

Altchinesische Medizin (Taoistische Medizin) kann mit der «Feuerschule» (Huo Shen) des Zheng Qin-An (18241911) assoziiert werden. Er hatte aufgrund seines taoistischen Studiums erkannt, dass es sich beim alten Klassiker Shang Han Lun (Abhandlung über Kälteschädigungen) von Zhang Zhongjing (150-219 n.Chr.) um ein taoistisches Werk handeln muss, das sich der stetigen Gefahr des natürlichen Yang-Verlustes beim Menschen und der daraus folgenden Kälteschädigungen bewusst war [11]. Die Welt ist kalt und dunkel, das Yang muss geschützt und gepflegt werden. Mangel an Yang führt zu Krankheitsanfälligkeit durch Geistesabwesenheit, Wehrschwäche (wei qi), Eindringen klimatischer Faktoren, Heilungsstörungen und Degeneration. Hauptschwächen sind von Geburt an angelegt (von gering bis ausgeprägt), zeigen sich bei Belastungen und Unfällen und entwickeln sich zu chronischen Schwächen und Krankheiten, wenn die richtigen therapeutischen und diätetischen Massnahmen unterbleiben - oder das Schicksal es will. Zunge, Puls, Ausstrahlung und vielfältige klinische Zeichen bestätigen oder modifizieren das Bild. Das Schicksal (und damit die alte Kosmologie von Him- 
melsstämmen und Erdenzweigen) ist ein wichtiger Bestandteil der Altchinesischen Medizin, wobei Krankheit immer auch Krise im Sinne einer Chance ist. Halten die Organkräfte (hält das Yang), kommt der Patient durch und kommt gereift und «integrierter» aus seiner Krankheit hervor (z.B. mit besserer Herz-Nieren-Koordination bzw. mit «dem Herzen am rechten Fleck»). Entscheidend für die Yin- oder Yang-Wirkung von (klassischen) Rezepturen ist die Dosierung der einzelnen Mittel. So muss das Blut kühl bleiben (wie das Öl im Motor); deshalb braucht es auch bei hochdosiertem Yang-Bedarf oft Yin-Mittel in der Rezeptur. Und Senken (Yin) kann das Steigen (Yang) unterstützen, so wie das fallende Wasser ein Wasserrad antreibt, das auf der Gegenseite ja eine steigende Bewegung vollführt. Deshalb ist etwa das kalte Gui Ban in der klassischen Rezeptur des Qian Yang Dan ein Mittel, das den Yang-Aufbau strategisch unterstützt und durch das Steigen und Senken überdies Herz und Niere zusammenführt (HerzNieren-Koordination). Pharmakologisches Denken nach moderner Art (3 Gramm Yang + 1 Gramm Yin = nur noch 2 Gramm Yang; also Yin weglassen, dann genügen 2 Gramm Yang) übersieht, wie es in der Natur zu- und her- und auf- und abgeht - oder wie erst Feuer und Wasser zusammen Dampf machen.

Klinische Erscheinungen sind gut zu ergründen. Herrscht Trockenheit (z.B. trockene Vaginalschleimhaut in der Menopause), dann mangelt eben das Feuer/Yang und nicht das Wasser/Yin. Wasser sinkt ja nach unten; erst genug Feuer macht Wasser zu Dampf und befeuchtet so bis nach oben. Die Naturbeobachtung gibt die Vorgabe, Bücher kann man falsch verstehen (in der neuzeitlichen Traditionellen Chinesischen Medizin (TCM) gilt trockene Schleimhaut in der Menopause als Yin-Mangel). Hin Chung Got, der uns diese Praxis gelehrt hat, ist Schüler von Tang Buqi (1917-2004), der die Schriften des oben genannten Zheng Qin-An herausgegeben hat. Die grosse klinische Bedeutung von Yang, Yang-Verlust bzw. Yang-Erhalt ist aus Sicht der Altchinesischen Medizin unübersehbar.

\section{Yang-Schwäche als möglicher Confounder}

Das Verdauungsfeuer ist eine Metapher für die Stoffwechselleistung. Für erfahrene Ärzte ist es nicht nur eine Metapher, sondern die klinisch sichtbare Kraft, welche die Kapazität von Verdauung und Stoffwechsel ausmacht. Es ist das Yang, in diesem Fall das Yang der Mitte/Milz. Die Leistung der Verdauung bzw. der Mitte ist nicht nur am Stuhlgang und allfälligen Verdauungsbeschwerden erkennbar, sondern auch etwa an der Fähigkeit, Sorgen und emotionale Einflüsse zu verdauen, sodass z.B. das Einschlafen nicht gestört wird. Starkes Yang bedeutet kräftige Verdauung, schwaches Yang heisst schwache Verdauung. In Wirklichkeit macht das Yang nicht an Organgrenzen halt, auch wenn man (didaktisch) von Milz-Yang, Nieren-Yang und Lungen-Yang spricht. Entweder ist Yang überall ausreichend vorhanden oder es fehlt überall, obwohl wir in der Diagnostik Schwerpunkte und graduelle Unterschiede erkennen. Schwaches Yang bedeutet schwächere Widerstandskraft - oder mit anderen Worten: grössere Anfälligkeit für Krankheiten.

Der ländliche Mensch in China, wie er in der China Study untersucht worden ist, ernährt sich traditionell so, wie er am besten überleben und sich am Leben freuen kann. Hat er wenig Yang/Kraft, isst er Fleisch, sooft er kann, weil dies die wichtigste Yang/Kraft-Quelle ist. Hat er genug Yang/Kraft, kann er am Fleischkonsum sparen. Hat er genug Yang/Kraft, so kann er aber auch Ballaststoffe gut verbrennen/verdauen und viel Gemüse (und Reis) essen. Er meidet aber möglichst Ballaststoff und Vollkorn, denn er will seine Verdauung nicht unnötig belasten. Hat er wenig Yang/Kraft, isst er zwar wie gesagt Fleisch, sooft er kann (Käse und Milchprodukte sind im ländlichen China unbekannt), aber er meidet Ballaststoffe, weil er diese mit seiner schwachen Verdauung nicht gut verdauen kann. Überdies weiss der ländliche Chinese traditionsgemäss, dass die Zubereitung und das Kochen entscheidend dafür sind, ob die Nahrung ihn stärkt. Er nimmt keine Rohkost $\mathrm{zu}$ sich (so wie dies auch in unseren Breiten nicht üblich war, solange die Menschen zum Arbeiten Kraft brauchten). Wenn diese traditionellen Auffassungen der chinesischen Ernährungslehre in Betracht gezogen werden, dann gilt der Konsum von Fleisch nicht als schädlich, sondern er stärkt vielmehr das Yang und damit die Abwehr von Krankheiten. So lässt sich also auf gutem Grund mutmassen, dass der Fleischkonsum bei Menschen mit Yang-Schwäche spontan grösser ist, wogegen Menschen mit viel Yang weniger Fleisch konsumieren, weil sie so Geld sparen und ihr Yin/Yang-Gleichgewicht vielleicht besser halten können. Nicht wegen des Fleischkonsums, sondern trotz des Fleischkonsums bekommen Menschen mit Yang-Schwäche häufiger Krankheiten. Korrelationen wie jene in der China Study lassen nie einen Schluss darüber zu, ob der untersuchte Nahrungsbestandteil gesund oder ungesund sei. Es kann wie gezeigt sogar das Gegenteil dessen vorgetäuscht werden, was wahr ist.

\section{Schlussfolgerungen}

Die statistische Korrelation zwischen Fleischverzehr und der Häufung von Krankheiten wie Herzinfarkt und Krebs in der China Study kann als Ergebnis statistischer Fehler und Trugschlüsse aufgefasst werden, die bekanntermassen auch das Gegenteil dessen «aufzeigen» können, was in Tat und Wahrheit richtig ist. Da die China Study nicht einmal Individuen erfasst bzw. innerhalb eines Landes verglichen hat, sondern lediglich (aggregierte) Durchschnittszahlen 
zwischen dem ländlichen China und den USA, ist die Gemüse-gegen-Fleisch-Hypothese unmöglich aus diesen Daten ableitbar, so wie es die Autoren anfänglich auch selbst erklärt hatten [1]. Denn Ernährung und Lebensstil in den USA haben sich wie in vielen industrialisierten Ländern weit von einer Yang-Pflege entfernt, wie sie im ländlichen China und in traditionellen Gesellschaften in der Regel besteht und auch in unseren Breiten bestanden hat, zumindest dort, wo nicht Armut dies gänzlich verhindert hat. Solange der Mensch Armut und grosse Entbehrungen überwinden und unter anderem genug Fleisch essen konnte, ist die Lebenserwartung überall und zu allen Zeiten gestiegen. Yang-Pflege besteht aus guter Ernährung und aus Qigong in allen Formen (d.h. Ruhe, Bei-sich-Sein, Gottesdienst, Kopf-leer-Machen, körperliche Anstrengung im Tempo des Qi und nur solange das Qi reicht, Verzicht auf übermässige Zerstreuung, Regelmässigkeit im Tagesablauf mit Schlaf zur rechten Zeit usw.). Zu grosse Hektik, Kopflastigkeit und ungünstige Ernährung (wie etwa «gesunde Ernährung» nach modernem Verständnis) sind der Anfang von Milz-Yang-Schwäche - je nach individueller Konstitution früher oder später im Leben. Ungeformter oder kraftloser Stuhlgang, Über- oder Untergewicht, degenerative Ablagerungen oder Gewebsschwäche und so weiter sind die Folge. Die erschöpfte Milz/Mitte verlangt nach immer mehr Fleisch oder Zucker, was nur gut geht, wenn die Ernährung ausgeglichen bleibt, das richtige Kochen das Yang optimiert und der Zuckerhunger dadurch nachlässt, so wie sich dies in der klinischen Praxis sehen lässt. Entscheidend ist, ob alle Zeichen der müden Milz überwunden und nicht zugedeckt werden. Rohkost, Joghurt, Vitamine und Nahrung ohne Yang (z.B. fleischlos) decken die Symptome zu, und man fühlt sich dann manchmal eine Zeit lang scheinbar besser. Wenn dann etwa eine zu starke Mens oder eine Inkontinenz noch stärker werden, zeigt sich die Sackgasse. Bei weit fortgeschrittenen Krankheiten kann es natürlich schwierig werden, das Yang noch $\mathrm{zu}$ «retten». Das «Kaltstellen» von Krankheitsäusserungen (auf der Yin-Ebene der sichtbaren Pathologie und der messbaren Pathophysiologie) kann dann einen Platz haben, auch wenn das Yang und damit der Geist beeinträchtigt werden. Die Taoistische Medizin zieht das Leben und Sterben mit einem wachen Geist vor.

Das «alte Wissen» über die natürliche Tendenz zum Yang-Verlust scheint im Übrigen universal und wird in vielen «alten Büchern» thematisiert - beispielsweise im Werk von Paracelsus, der vom «Licht der Natur» spricht und im 16. Jahrhundert die mittelalterliche Hochscholastik der früheren Blütezeit noch einmal vor ihrer Verschulung durch die spätscholastischen Universitäten retten wollte. Seine Verteidigungsreden kurz vor seinem Tod stimmen mit den Auffassungen der Altchinesischen Medizin in frappanter Weise überein.

In der traditionellen chinesischen Ernährungslehre steht das ausgewogene Mass im Vordergrund; es gibt im Grunde keine gesunde oder ungesunde Nahrung an sich, sondern es kommt darauf an: auf die Reihenfolge (Suppe und nicht roher Salat am Anfang), auf die Jahreszeit, auf die Tageszeit, auf die individuelle Konstitution und auf den Ort (Zeit, Ort und Person). Und es gibt immer Ausnahmen, die die Regel bestätigen. Eine echte Studie über die Ernährung in China würde die Thesen der sogenannten China Study nie hervorbringen. Diese sind eine Kopfgeburt aus dem urbanen Westen. Die Yang-Mangel-Hypothese lässt sich andererseits nicht einfach über Labormessungen nachweisen, denn der Ausdruck von Yang hängt von wandelnden Umständen ab und ist nur dem geschulten und wachen Auge des Beobachters ersichtlich. Sie ist mit unserem modernen klinischen Instrumentarium nicht fassbar, ausser indirekt über eine genaue Anamnese der Leistungsfähigkeit der Organe. Eine entscheidungsanalytische Orientierung an der Aussagekraft klinischer Information - vereint mit einem genauen Verständnis von Sensitivität und Prädiktivität von apparativer Information im Vergleich zu klinisch-anamnestischer Information - ist bereits von hervorragenden Schulmedizinern formuliert [12-14]. Dies wird das Yang, d.h. die Widerstandskräfte, im Prinzip auch der modernen klinischen Wissenschaft zugänglich machen - und sie wird es brauchen. Das technisch hochentwickelte Datensammeln der modernen «Wissensgesellschaft» reicht nicht. Es gilt zu erkennen, dass es letztlich den geübten klinischen Blick braucht, um vor lauter Bäumen den Wald (und das Yang) zu sehen.

\section{Disclosure Statement}

Der Autor hat für diesen Artikel keinerlei Unterstützungen erhalten Ein Interessenkonflikt besteht nicht.

\section{Literatur}

1 Campbell TC, Parpia B, Chen J: Diet, lifestyle, and the etiology of coronary artery disease: the Cornell China Study. Am J Cardiol 1998;82(suppl 2):18-21.
2 Schmidt JG, Bucher HC, Steurer J: Zeitgemässe Beurteilung medizinischen Wissens - Kursskriptum 1999. Stiftung Paracelsus heute,Einsiedeln.www.paracelsus-heute.ch/cms/ stiftung/methodik_studien/Kursskriptum.pdf.
3 Abel U, Koch A (eds): Non-randomized comparative clinical studies. Düsseldorf, Symposium Publishing, 1998. 
4 Schmidt JG: Die Vision einer pragmatischen klinischen Forschung oder das Ende der Diskussion über «Placebo» und «spezifische Wirkungen»; in Schmidt JG (ed): Placebo - Wertvoll wenn es dem Patienten nützt? Methodologie einer nutzenorientierten, pragmatischen klinischen Forschung. Forsch Komplementärmed 1998;5(suppl 1):1-139.

5 The Cardiac Arrhythmia Suppression Trial (CAST) Investigators: Preliminary report: effect of encainide and flecainide on mortality in a randomized trial of arrhythmia suppression after myocardial infarction. N Engl J Med 1989;321:406-412.

6 Rossouw JE, Anderson GL, Prentice RL, LaCroix AZ, Kooperberg C, Stefanick ML, Jackson RD, Beresford SA, Howard BV Johnson KC, Kotchen JM, Ockene J; Writing Group for the Women's Health Initiative Investigators: Risks and benefits of estrogen plus progestin in healthy postmenopausal women - principal results from the Women's Health Initiative randomized controlled trial. JAMA 2002;288:321-333.
7 Stiftung Paracelsus heute: Osteoporose: Benötigen Frauen nach den Wechseljahren Hormone? Patient sein heute - Medizin auf dem Prüfstand kritischer Wissenschaft, ed 2. 1995.

8 Hemminki E, McPherson K: Impact of postmenopausal hormone therapy on cardiovascular events and cancer: pooled data from clinical trials. BMJ 1997;315:149.

Miller ER 3rd, Pastor-Barriuso R, Dalal D, Riemersma RA, Appel LJ, Guallar E: Meta-analysis: high-dosage vitamin E supplementation may increase all-cause mortality. Ann Int Med 2005;142:37-46.

10 Omenn GS, Goodman GE, Thornquist MD, Balmes J, Cullen MR, Glass A, Keogh JP, Meyskens FL, Valanis B, Williams JH, Barnhart S, Hammar S: Effects of a combination of beta carotene and vitamin A on lung cancer and cardiovascular disease. N Engl J Med 1996;334:1150-1155.
11 Neeb G: Vom Füllen der Leere - Yang: Das Feuer des Lebens. Schiedlberg, Bacopa, 2011.

12 Sox HC, Blatt MA, Higgins HC, Marton KI: Medical Decision-Making. Boston, ButterworthHeinemann, 1988.

13 Feinstein AR: Clinimetrics. New Haven, Yale University Press, 1987.

14 Feinstein AR: Das irreführende Credo harter Daten in der heutigen klinischen Praxis - das Problem sinnvoller klinischer Erfolgskriterien. Forsch Komplementärmed 1994;1:260-265. 